\title{
Application of symmetry-adapted functions method for three-dimensional reconstruction of biological macromolecules with dihedral symmetry
}

\author{
Songjun Zeng ${ }^{1,2}$, Hongrong Liu ${ }^{1,2}$, Qibin Yang ${ }^{1,2}$ \\ 1. Institute of Modern Physics, Xiangtan University, Xiangtan, China. 2. Faculty of Materials, Optoelectronics and Physics, \\ Xiangtan University, Xiangtan, China \\ Corresponding: Qibin Yang. Address: Faculty of Materials, Optoelectronics and Physics, Xiangtan University, Xiangtan \\ 411105, China. Telephone: 86-0732-8292-113. E-mail: yqb@xtu.edu.cn
}

Received: September 28, 2011

Accepted: November 9, 2011

Published: December 1, 2011

DOI : $10.5430 / j b g c . v 1 n 1 p 33$

URL: http://dx.doi.org/10.5430/jbgc.v1n1p33

\begin{abstract}
Three-dimensional (3D) structure reconstruction of single particles has played an important role in understanding the mechanisms of macromolecular assembly. We demonstrate a Dihedral Symmetry-adapted function method (DSAF) to implement 3D reconstruction of macromolecular complexes with dihedral symmetry. A set of formulations for 3D reconstruction of biological objects with dihedral symmetry is presented in detail. To verify the feasibility and efficiency of the DSAF method for dihedral symmetrical objects, two special illustrations of simulation data with D6, D7 symmetry, were taken as examples to implement reconstruction by the DSAF method. The results show that the DSAF method is robust and suppresses noise in the three-dimensional reconstruction of dihedral symmetrical objects at high resolution. Thus the DSAF method is suitable for reconstruction of biological complexes with various kinds of dihedral symmetry.
\end{abstract}

\section{Key words}

Dihedral symmetry-adapted functions, Three-dimensional structure, Dihedral symmetry

\section{I ntroduction}

Three-dimensional (3D) structure reconstruction is rapidly becoming an indispensable tool for understanding the properties and functions of macromolecular complexes and biological nanomachines. Especially with the recent development of single particle reconstruction, cryo-electron microscopy (Cryo-EM) has played an increasingly important role in determining 3D structures down to sub-nanometer resolution, even near-atomic resolution [1-6]. The determination of the structures of macromolecular complexes by Cryo-EM techniques consists of two essential steps: the determination of orientation and center parameters, and 3D reconstruction. Structure refinement should be carried out gradually, aiming at pushing toward higher resolution by repeating these two steps. Various approaches for 3D reconstruction have been developed including the Fourier-Bessel synthesis method [7], the direct Fourier inversion method [8, 9], and the symmetry-adapted functions method (SAF) [10-12]. Among of these approaches, the SAF method can most efficiently utilize the intrinsic symmetry of the objects. Crowther et al. pointed out in their seminal paper that using SAF to interpolate 
the Fourier space of a virus would make efficient use of the available data [7]. Provencher and Vogel presented a method of 3D reconstruction by symmetry-adapted functions and applied this method to reconstruct simulated model structures and biological objects $[13,14]$. Zheng et al. used a spherical harmonics method to determine the structure of viruses from solution X-ray scattering data [15]. Navaza [10] systematically developed formulations for 3D reconstruction of icosahedral viruses including ab initio determination of the origins and orientations of particles and interpolation of data in the reciprocal space by the icosahedral symmetry-adapted function (ISAF) method. Our recent work [11] showed that the ISAF method could enhance resolution compared with the Fourier-Bessel method when it was applied to the same particles. We believe that the ISAF method has good future prospects because it efficiently utilizes the symmetry of objects. Macromolecular assemblies have various symmetries, such as icosahedral, octahedral, tetrahedral, and dihedral symmetry. Following the great successes in solving the icosahedral shells of many viruses at sub-nanometer resolution, attempts have been made to study non-icosahedral components with low order symmetry. Many software packages which adopt the direct Fourier inversion method such as EMAN [8], FREALIGN [9] have been successfully used for reconstructing 3D structures of biological objects with dihedral symmetry at sub-nanometer resolutions; notable examples are the auxilin-bound clathrin coat structure with D6 symmetry solved at $12 \AA$, and then extended to $8 \AA$ resolution [16], the GroEL structure with D7 symmetry revealed at $6 \AA$ [6] and recently at near-atomic resolution of $4.2 \AA$ [17], the 20S proteasome structure with D7 symmetry solved at $6.8 \AA$ resolution [18], and keyhole limpet hemocyanin isoform 1 (KLH1) with an overall D5 point-group symmetry reconstructed at $11.3 \AA$ resolution [19]. However, there are no existing reports of 3D reconstruction of biological objects with dihedral symmetry using the SAF method. At this stage, Our previous work have established icosahedral symmetry-adapted functions method (ISAF) [11] and octahedral symmetry-adapted functions method (OSAF) [12] for 3D reconstruction of macromolecular complexes with icosahedral and octahedral symmetry respectively. In this article we attempt to extend symmetry-adapted functions method for 3D reconstruction of macromolecular assemblies with all dihedral symmetry. To verify the feasibility and advantages of this approach, we performed 3D reconstruction of two known structures-auxilin-bound clathrin coat with D6 symmetry (1Xi4.pdb) [16] and GroEL (3Cau.pdb) [17] with D7 symmetry-downloaded from the protein data bank (PDB), by the DSAF method using simulated data. The results demonstrate that the DSAF method is suitable for all dihedral symmetrical objects and can retrieve the 3D structures of objects with dihedral symmetry at high resolution.

\section{Method}

\subsection{Dihedral symmetry-adapted functions}

In this section we give the formulae and definitions which are required to determine the DSAF. Because the symmetry-adapted functions (SAFs) are linear combinations of the spherical harmonics functions $Y_{l, m}(\theta, \varphi)$ [20], the DSAF can be written as:

$$
D_{l, \mu}(\theta, \varphi)=\sum_{m=-L}^{L} C_{l, m}^{\mu} Y_{l, m}(\theta, \varphi)
$$

where $C_{l, m}^{\mu}$ is an expansion coefficient, and $m$ is a multiple of $n$ because the Z-axis is parallel to the $n$-fold axis for $D n$ symmetry. $Y_{l, m}(\theta, \varphi)$ is the normalized spherical harmonic functions and can be described as 


$$
Y_{l, m}(\theta, \varphi)=N_{l, m} P_{l}^{m}(\cos \theta) e^{i m \varphi}
$$

where $P_{l}^{m}(\cos \theta)$ is the associated Legendre function and $N_{l, m}$ denotes a normalization coefficient which is identical to $\sqrt{\frac{(2 l+1)}{4 \pi} \frac{(l-|m|) !}{(l+|m|) !}}$.

$\mu(l)$ is the multiplicity for a given order $l$, and can be obtained by the following formulae for any dihedral symmetry (Dn):

$$
\begin{array}{ll}
\mu(l)=\operatorname{int}(l / n)+1 & \text { for } l=\text { even } \\
\mu(l)=\operatorname{int}(l / n) & \text { for } l=\text { odd }
\end{array}
$$

where int $(l / n)$ indicates the integer part of $l / n$.

Now the major problem is finding the coefficients $C_{l, m}^{\mu}$ of the DSAF (see expression (1)). The dihedral groups are the simplest non-Abelian point groups and many numerical solutions for the dihedral groups have been implemented [21, 22]. In this paper, we adopted the algebraic method for calculating the coefficients $C_{l, m}^{\mu}$ of the DSAF, which is suitable for all dihedral groups [23]. According to the method mentioned above, the DSAF can be directly written as the following expressions.

$$
\begin{aligned}
& m \neq 0 \quad D_{l, \mu}(\theta, \varphi)=C_{l, m}^{\mu}\left[Y_{l m}(\theta, \varphi)+(-1)^{l} Y_{l-m}(\theta, \varphi)\right] \\
& m=0\left\{\begin{array}{l}
l=\text { even } \rightarrow C_{l, 0}^{\mu}=1, D_{l, \mu}(\theta, \varphi)=Y_{l, 0}(\theta, \varphi) \\
l=\text { odd } \rightarrow C_{l, 0}^{\mu}=0
\end{array}\right.
\end{aligned}
$$

where $C_{l, m}^{\mu}$ is identical to a constant $\sqrt{2} / 2$ in Eq. (4.1)

To aid understanding of the DSAF, we take D6 and D7 symmetry as examples. For D6 symmetry, according to Eq. (1), Eq. (2), Eq. (3.1), Eq. (3.2), Eq. (4.1), and Eq. (4.2), the DSAF for $l=25$ and $l=26$ can be obtained as follows.

When $l=25, \mu(25)=\operatorname{int}(25 / 6)=4$

$$
\begin{aligned}
& D_{25,1}(\theta, \varphi)=\frac{\sqrt{2}}{2}\left[Y_{25,6}(\theta, \varphi)-Y_{25,-6}(\theta, \varphi)\right]=i \sqrt{2} N_{25,6} P_{25}^{6}(\cos \theta) \sin (6 \varphi) \\
& D_{25,2}(\theta, \varphi)=\frac{\sqrt{2}}{2}\left[Y_{25,12}(\theta, \varphi)-Y_{25,-12}(\theta, \varphi)\right]=i \sqrt{2} N_{25,12} P_{25}^{12}(\cos \theta) \sin (12 \varphi)
\end{aligned}
$$




$$
\begin{aligned}
& D_{25,3}(\theta, \varphi)=\frac{\sqrt{2}}{2}\left[Y_{25,18}(\theta, \varphi)-Y_{25,-18}(\theta, \varphi)\right]=i \sqrt{2} N_{25,18} P_{25}^{18}(\cos \theta) \sin (18 \varphi) \\
& D_{25,4}(\theta, \varphi)=\frac{\sqrt{2}}{2}\left[Y_{25,24}(\theta, \varphi)-Y_{25,-24}(\theta, \varphi)\right]=i \sqrt{2} N_{25,24}{ }_{25}^{24}(\cos \theta) \sin (24 \varphi)
\end{aligned}
$$

When $l=26, \mu(26)=\operatorname{int}(26 / 6)+1=5$

$$
\begin{aligned}
& D_{26,1}(\theta, \varphi)=Y_{26,0}(\theta, \varphi)=N_{26,0} P_{26}^{0}(\cos \theta) \\
& D_{26,2}(\theta, \varphi)=\frac{\sqrt{2}}{2}\left[Y_{26,6}(\theta, \varphi)-Y_{26,-6}(\theta, \varphi)\right]=\sqrt{2} N_{26,6} P_{26}^{6}(\cos \theta) \cos (6 \varphi) \\
& D_{26,3}(\theta, \varphi)=\frac{\sqrt{2}}{2}\left[Y_{26,12}(\theta, \varphi)-Y_{26,-12}(\theta, \varphi)\right]=\sqrt{2} N_{26,12} P_{26}^{12}(\cos \theta) \cos (12 \varphi) \\
& D_{26,4}(\theta, \varphi)=\frac{\sqrt{2}}{2}\left[Y_{26,18}(\theta, \varphi)-Y_{26,-18}(\theta, \varphi)\right]=\sqrt{2} N_{26,18} P_{26}^{18}(\cos \theta) \cos (18 \varphi) \\
& D_{26,5}(\theta, \varphi)=\frac{\sqrt{2}}{2}\left[Y_{26,24}(\theta, \varphi)-Y_{26,-24}(\theta, \varphi)\right]=\sqrt{2} N_{26,24} P_{26}^{24}(\cos \theta) \cos (24 \varphi)
\end{aligned}
$$

For D7 symmetry,, the DSAF for $l=25$ and $l=26$ can be obtained by the same method.

When $l=25, \mu(25)=\operatorname{int}(25 / 7)=3$

$$
\begin{aligned}
& D_{25,1}(\theta, \varphi)=\frac{\sqrt{2}}{2}\left[Y_{25,7}(\theta, \varphi)-Y_{25,-7}(\theta, \varphi)\right]=i \sqrt{2} N_{25,7} P_{25}^{7}(\cos \theta) \sin (7 \varphi) \\
& D_{25,2}(\theta, \varphi)=\frac{\sqrt{2}}{2}\left[Y_{25,14}(\theta, \varphi)-Y_{25,-14}(\theta, \varphi)\right]=i \sqrt{2} N_{25,14} P_{25}^{14}(\cos \theta) \sin (14 \varphi) \\
& D_{25,3}(\theta, \varphi)=\frac{\sqrt{2}}{2}\left[Y_{25,21}(\theta, \varphi)-Y_{25,-21}(\theta, \varphi)\right]=i \sqrt{2} N_{25,21} P_{25}^{21}(\cos \theta) \sin (21 \varphi)
\end{aligned}
$$

When $l=26, \mu(26)=\operatorname{int}(26 / 7)+1=4$

$$
\begin{aligned}
& D_{26,1}(\theta, \varphi)=Y_{26,0}(\theta, \varphi)=N_{26,0} P_{26}^{0}(\cos \theta) \\
& D_{26,2}(\theta, \varphi)=\frac{\sqrt{2}}{2}\left[Y_{26,7}(\theta, \varphi)-Y_{26,-7}(\theta, \varphi)\right]=\sqrt{2} N_{26,7} P_{26}^{7}(\cos \theta) \cos (7 \varphi)
\end{aligned}
$$




$$
\begin{aligned}
& D_{26,3}(\theta, \varphi)=\frac{\sqrt{2}}{2}\left[Y_{26,14}(\theta, \varphi)-Y_{26,-14}(\theta, \varphi)\right]=\sqrt{2} N_{26,14} P_{26}^{14}(\cos \theta) \cos (14 \varphi) \\
& D_{26,4}(\theta, \varphi)=\frac{\sqrt{2}}{2}\left[Y_{26,21}(\theta, \varphi)-Y_{26,-21}(\theta, \varphi)\right]=\sqrt{2} N_{26,21} P_{26}^{21}(\cos \theta) \cos (21 \varphi)
\end{aligned}
$$

In fact, one can obtain real functions from DSAF by combining two complex conjugate functions with $m$ values of opposite sign. Then the DSAF can be divided into two parts

$$
D_{l, \mu}(\theta, \phi)=D_{l, \mu}^{c}(\theta, \phi)+i D_{l, \mu}^{s}(\theta, \phi)
$$

where $D_{l, \mu}^{c}(\theta, \phi)$ and $D_{l, \mu}^{s}(\theta, \phi)$ denote real and imaginary parts respectively

when $l$ is even,

$$
\begin{cases}m \neq 0 & \mathrm{D}_{l, \mu}^{c}(\theta, \varphi)=2 C_{l, m}^{\mu} P_{l}^{m}(\cos \theta) \cos m \varphi \\ m=0 & \mathrm{D}_{l, \mu}^{c}(\theta, \varphi)=C_{l, m}^{\mu} P_{l}^{m}(\cos \theta) \cos m \varphi\end{cases}
$$

When $l$ is odd,

$$
\begin{cases}m \neq 0 & \mathrm{D}_{l, \mu}^{s}(\theta, \varphi)=2 C_{l, m}^{\mu} P_{l}^{m}(\cos \theta) \sin m \varphi \\ m=0 & \mathrm{D}_{l, \mu}^{s}(\theta, \varphi)=0\end{cases}
$$

Therefore, one may use the $D_{l, \mu}^{c}(\theta, \varphi)$ to fit the real part of the structure factor and $D_{l, \mu}^{s}(\theta, \varphi)$ the imaginary part of the structure factor of biological complexes with dihedral symmetry in reciprocal space.

\subsection{Procedures for 3D reconstruction by the DSAF method}

It is well known that the 3D structure of objects can be described as the potential function which is determined by inverse Fourier transform of the structure factors; its expression in spherical coordinates is presented as follows:

$$
\rho(\boldsymbol{r})=\int F(\boldsymbol{R}) \exp (-2 \pi i \boldsymbol{R} \cdot \boldsymbol{r}) R^{2} \sin \Theta d R d \Theta d \Phi
$$

where $\boldsymbol{r}$ and $\boldsymbol{R}$ denote the vectors in real and Fourier spaces respectively. 
The commonly used method for 3D reconstruction is the Fourier-Bessel method [7], where the exponential function $\exp \left(\operatorname{in}\left(\phi+\frac{\pi}{2}\right)\right)$ is taken as a basic function to fit data in the reciprocal space. Compared to Crowther's Fourier-Bessel method, the DSAF is used as a basic function to interpolate the structure factor $F(\boldsymbol{R})$ in the reciprocal space of spherical coordinates in this paper, which is described as following formula.

$$
F(\boldsymbol{R})=F(R, \Theta, \Phi)=\sum_{l=0}^{\infty} \sum_{\mu=1}^{n_{l}} f_{l, \mu}(R) D_{l, \mu}(\Theta, \Phi)
$$

where $f_{l, \mu}(R)$ is the expansion coefficient, whose value depends on the Fourier radius $R$ of a spherical shell, and $n_{l}$ is the multiplicity.

According to Eq. (8), to find the coefficients $f_{l, \mu}(R)$, one should use all the data on the surface of a sphere with radius=R.

Therefore, the complex function of structure factors is described as the sum of even and odd real DSAF according to the Eqs. (6a) and (6b).

$$
\begin{aligned}
& F_{r}(R, \Theta, \Phi)=\sum_{l=0(\bmod 2)}^{\infty} \sum_{\mu=1}^{n_{L}} f_{\text {leven }, \mu}(R) D_{l, \mu}^{c}(\Theta, \Phi) \\
& i F_{i}(R, \Theta, \Phi)=i \sum_{l=1(\bmod 2)}^{\infty} \sum_{\mu=1}^{n_{l}} f_{\text {lodd }, \mu}(R) D_{l, \mu}^{s}(\Theta, \Phi)
\end{aligned}
$$

where $F_{r}(R, \Theta, \Phi)$ and $F_{i}(R, \Theta, \Phi)$ denote the real and imaginary parts of $F(R, \Theta, \Phi)$ respectively.

The potential function can be obtained by substituting Eq. (9) and Eq. (10) into Eq. (7).

$$
\begin{aligned}
\rho(r, \theta, \varphi)=4 \pi[ & \sum_{l=0 \bmod 2}^{\infty}(-i)^{l} \sum_{\mu=1}^{n_{L}}\left(\int_{0}^{\infty} f_{\text {leven }, \mu}(R) j_{l}(2 \pi R r) R^{2} d R\right) D_{l, \mu}(\theta, \varphi)+ \\
& \left.\sum_{l=1 \bmod 2}^{\infty}(-i)^{l} \sum_{\mu=1}^{n_{L}}\left(\int_{0}^{\infty} f_{\text {lodd }, \mu}(R) j_{l}(2 \pi R r) R^{2} d R\right) D_{l, \mu}(\theta, \varphi)\right]
\end{aligned}
$$


where $j_{l}(2 \pi R r)$ labels the spherical Bessel functions, and its recurrence relationship can be seen in reference [24].

The 3D reconstruction by DSAF can be implemented in the following five steps.

- Determine the orientation and center parameters of each particle by means of common lines or matching computed projections avenues.

- Calculate the DSAFs by Eq. (4.1) and Eq. (4.2) up to the required order.

- Set up two linear equation groups according to Eq. (9) and Eq. (10) in terms of structure factors determined by the Fourier transform of the data of the experimentally measured images.

- Obtain the fitting coefficients $f_{\text {even, } \mu}(R)$ and $f_{\text {odd }, \mu}(R)$ by solving the two linear equation groups through the least squares method.

- $\quad$ Reconstruct the 3D structures of objects with dihedral symmetry using Eq. (11).

\section{Results and discussion}

In this section, to test the robustness of the DSAF method, we took the auxilin-bound clathrin coat with D6 symmetry (1Xi4.pdb) [16] and GroEL with D7 symmetry (3Cau.pdb) [17] as examples, and downloaded their structures from the Protein Data Bank (PDB). Simple projection/reconstruction tests of the two simulated datasets with various levels of added noise were performed by the DSAF method in the following way. First, the simulated 3D density maps of auxilin-bound clathrin coat and GroEL without noise were generated at a resolution of $4.5 \AA$ and $4.0 \AA$ using pdb2mrc from EMAN' s procedure library [8]. Then based on these 3D density maps, two thousand projections with random orientations were generated using the real-space projection method with trilinear interpolation by using EMAN's project3d procedure for both models. Thereafter random noise was added to each projection at different levels of signal-to-noise ratio (S/N) such as $\mathrm{S} / \mathrm{N}=0.1,0.5$ and 0.8. Fig.1 and Fig. 2 show three representative projections with $\mathrm{S} / \mathrm{N}$ $=0.1,0.5$ and 0.8 and without noise, for the clathrin coat model and the GroEL model respectively. The structures become obscure as the $\mathrm{S} / \mathrm{N}$ decreases and we cannot even find any structures when $\mathrm{S} / \mathrm{N}$ is equal to 0.1 . Finally, 3D reconstructions of the two models using a total of 2000 projections (with three different $\mathrm{S} / \mathrm{N}$ ratios) with predefined Euler angles and center parameters were performed at high resolution with the DSAF method. Fig. 3 and Fig. 4 show the reconstructed results of the clathrin coat model and the GroEL model respectively. It can be seen from Fig. 3 and Fig. 4 that the reconstructed structures with different $\mathrm{S} / \mathrm{N}$ are in good agreement with the standard models, although those with $\mathrm{S} / \mathrm{N}=0.1$ lose considerable detail. Fig. 5 presents 2D sections of the standard and reconstructed results for the GroEL model with different S/Ns viewing along the 7-fold axis. Fig. 6 shows 2D sections of the standard model and reconstructed results of the clathrin coat with different S/Ns viewing along the 6-fold axis. From the 2D slice maps of both Fig. 5 and Fig. 6, we can see that the reconstructed results of both models with different $\mathrm{S} / \mathrm{Ns}$ are all similar to the original structures, despite some noise in the reconstructed results. As it is well known, a perfect reconstruction is impossible with such high levels of added noise. That is to say, 3D reconstruction by the DSAF method for objects with dihedral symmetry is feasible and robust. For quantitative comparison, the Fourier Shell Correlations (FSC) between the standard models and the reconstructed structures [25, 26] were calculated and are presented in Fig. 3e and Fig. 4e as functions of spatial frequency. According to the FSC $=0.5$ criterion, the nominal resolutions as shown in Fig.3e of the reconstructed results for GroEL with $\mathrm{S} / \mathrm{N}=0.1,0.5$ and 0.8 are approximately $5.7 \AA, 4.4 \AA$ and $4.0 \AA$, respectively. In the clathrin coat model, the nominal resolutions are $8.3 \AA, 4.7 \AA$ and $4.5 \AA$ for $\mathrm{S} / \mathrm{N}=0.1,0.5$ and 0.8 , respectively. Thus the FSC curves show that the 
reconstructed results with $\mathrm{S} / \mathrm{N}=0.8$ and 0.5 have almost the same nominal resolution as the standard structures. The resolution for $\mathrm{S} / \mathrm{N}=0.1$ is much worse. Naturally, with very low $\mathrm{S} / \mathrm{N}$, more particles are needed to achieve high resolution.

Figure 1. Typical projections of the clathrin coat model: (a) three typical projections without noise. (b) with $\mathrm{S} / \mathrm{N}=$ 0.8 , (c) with $\mathrm{S} / \mathrm{N}=0.5$, (d) with $\mathrm{S} / \mathrm{N}=0.1$

Figure 2. Typical projections of the GroEL model: (a) three typical projections without noise, (b) with $\mathrm{S} / \mathrm{N}=0.8$,

a

b

c

(c) with $\mathrm{S} / \mathrm{N}=0.5$, (d) with $\mathrm{S} / \mathrm{N}=0.1$





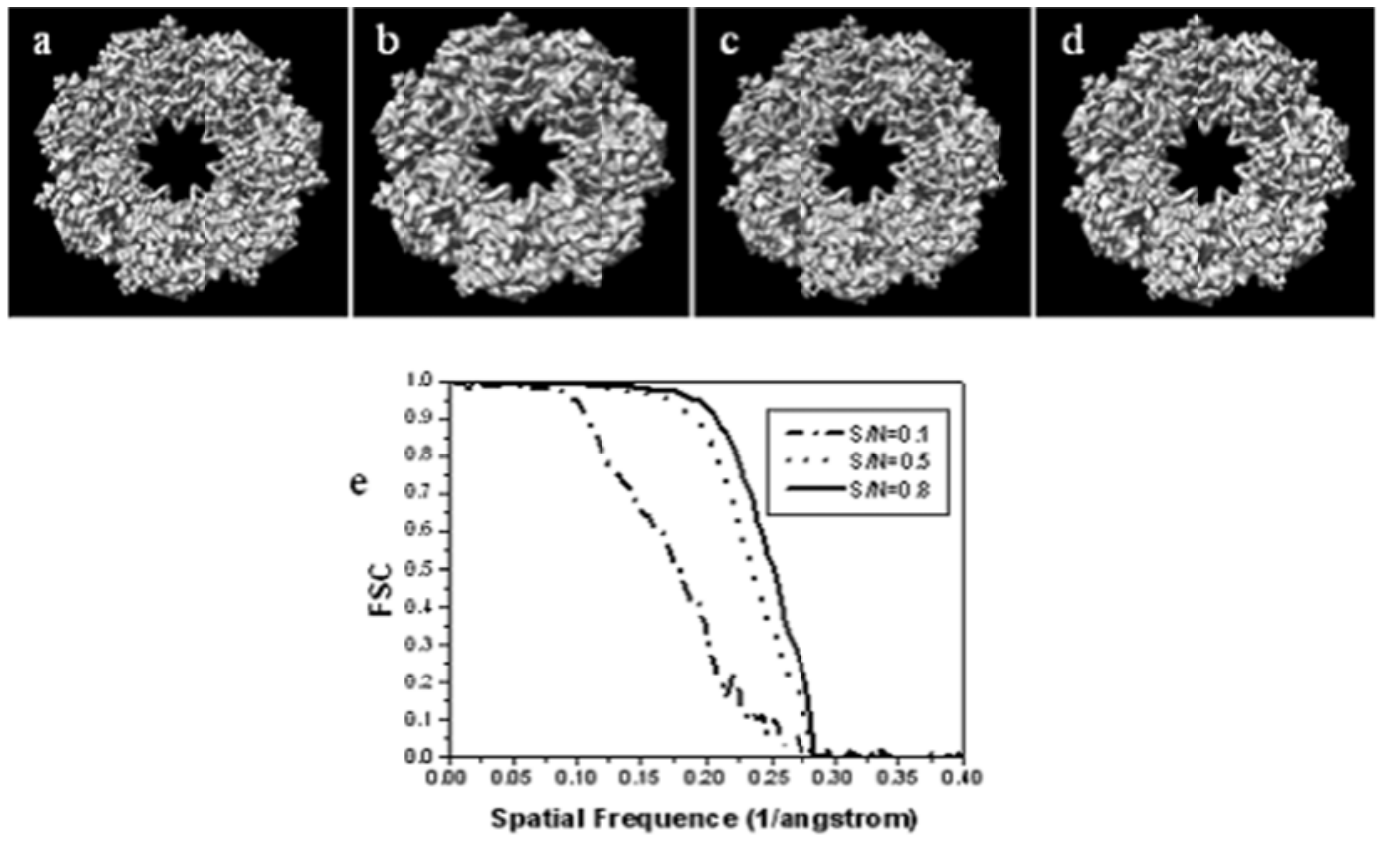

Figure 3. The reconstructed structures of GroEL viewed along the 7-fold axis: (a) the standard 3D structures, (b) the reconstructed 3D structure with $\mathrm{S} / \mathrm{N}=0.1$, (c) $\mathrm{S} / \mathrm{N}=0.5$, (d) $\mathrm{S} / \mathrm{N}=0.8$, (e) FSC curves at the three different noise level
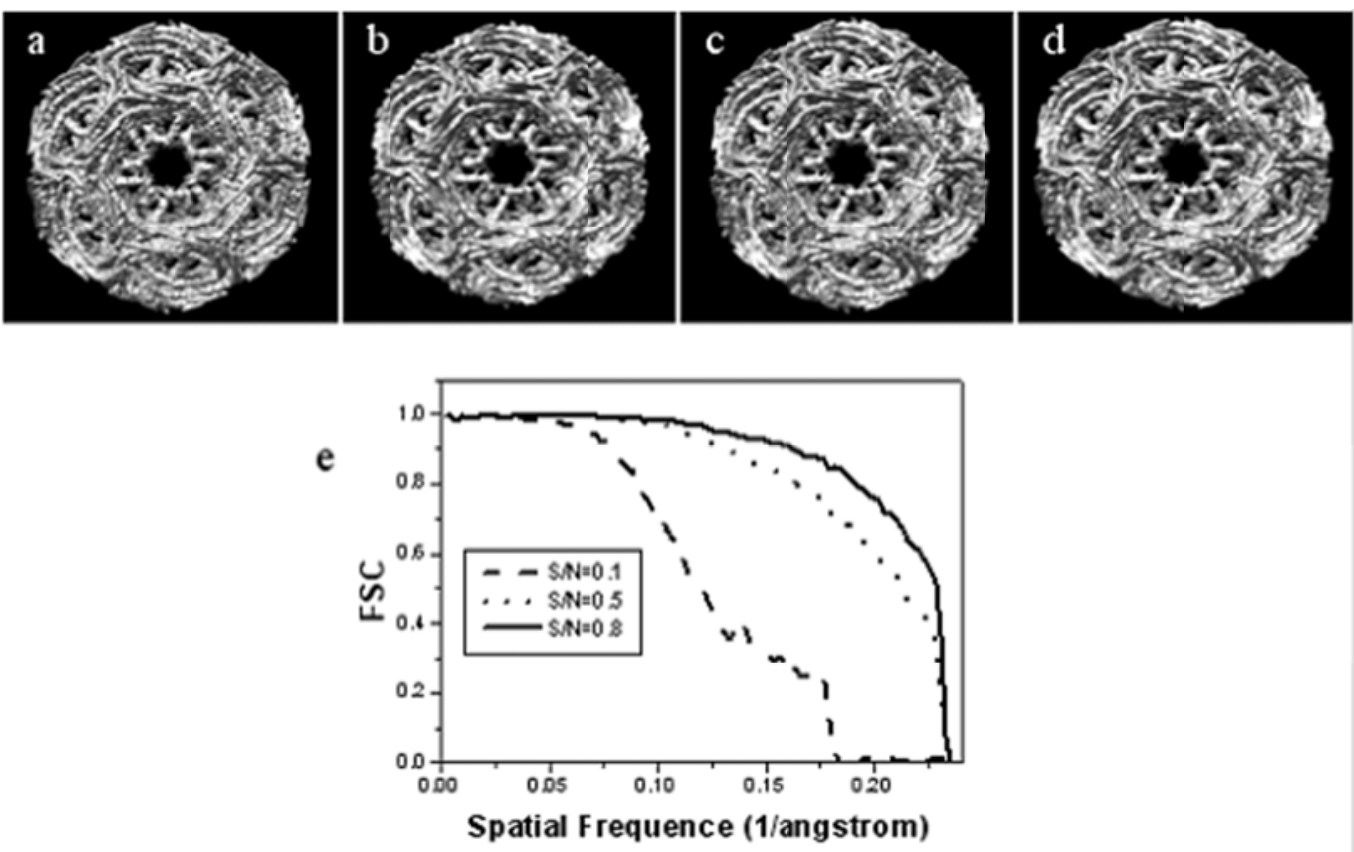

Figure 4. The reconstructed structures of the clathrin coat viewed along the 6-fold axis: (a) the standard 3D structures, (b) the reconstructed 3D structure with $\mathrm{S} / \mathrm{N}=0.1$, (c) $\mathrm{S} / \mathrm{N}=0.5$, (d) $\mathrm{S} / \mathrm{N}=0.8$, (e) FSC curves at the three different noise level 

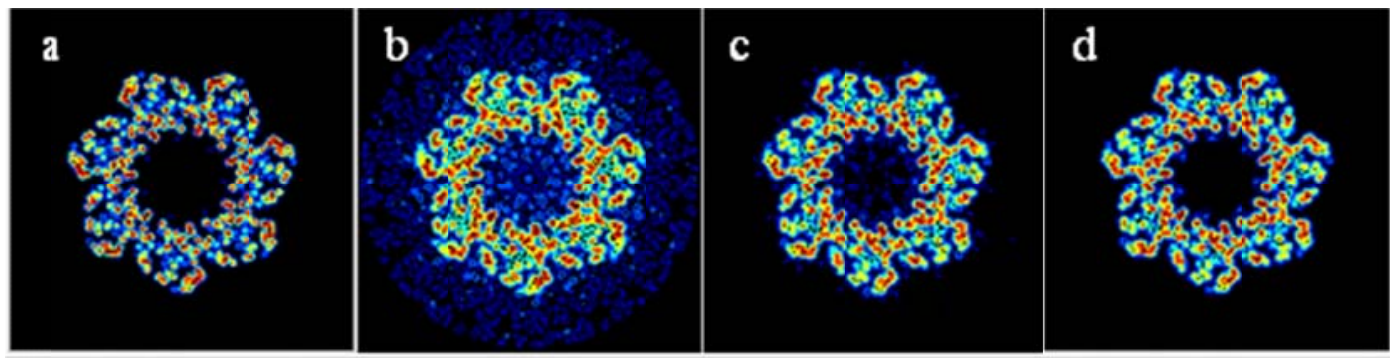

Figure 5. Two-dimensional sections of the standard and DSAF-reconstructed structures of the GroEL viewing along the 7-fold axis: (a) the standard model, (b) the reconstructed result with $\mathrm{S} / \mathrm{N}=0.1$, (c) $\mathrm{S} / \mathrm{N}=0.5$, (d) $\mathrm{S} / \mathrm{N}=0.8$


Figure 6. Two-dimensional sections of the standard and DSAF-reconstructed structures of clathrin coat viewing along the 6-fold axis: (a) the standard model, (b) the reconstructed results with $\mathrm{S} / \mathrm{N}=0.1$, (c) $\mathrm{S} / \mathrm{N}=0.5$, (d) $\mathrm{S} / \mathrm{N}=0.8$

\section{Conclusions}

In this article, a new DSAF method has been proposed for 3D reconstruction of macromolecular complexes with dihedral symmetry. A set of formulations was derived. To verify its feasibility and ability to reduce the influence of noise for macromolecules with dihedral symmetry, 3D reconstructions of two models such as GroEL and clathrin coat, with D7 and D6 symmetry at three different $\mathrm{S} / \mathrm{N}$ ratios $(\mathrm{S} / \mathrm{N}=0.1,0.5$ and 0.8 ) had been carried out using the DSAF method. The reconstructed results showed that the DSAF method can achieve very high resolution for both D6 and D7 symmetrical objects even when $\mathrm{S} / \mathrm{N}$ is equal to 0.1 . Thus the DSAF method is suitable for all kinds of dihedral symmetry (Dn) and can attain high resolution even with very low $\mathrm{S} / \mathrm{N}$.

It should be noted that in these simulations, the orientations and center parameters were predetermined, but in practice 3D structures would be reconstructed from experimentally measured data with unknown centers and orientations. In this case, one must first determine the center and orientation of a projection. The DSAF method is currently developed only for the 3D reconstruction step in Cryo-EM image processing; another program such as EMAN, FREALIGN, etc. must be used first to determine the orientation and center parameters of a particle, before adopting the DSAF method for 3D reconstruction. The DSAF method for 3D reconstruction is still in early development; there is plenty of scope for improving the program. We believe that this new method has a promising future. A reconstruction with experimental data based on the principle described above is proceeding and will be reported later.

\section{Acknowledgements}

This work is supported by the National Natural Science Foundation of China (No.10874144 and No. 31070663), the Scientific Foundation of Education Department of Hunan Province (No. 08C885) and the National Basic Research Program of China (No. 2010CB912400). 


\section{References}

[1] Bottcher B., Wynne SA, Crowther RA. Determination of the fold of the core protein of hepatitis B virus by electron cryomicroscopy. Nature. 1997;386:88-91. PMid: 18206961.

[2] Conway JF, Cheng N, Zlotnick A, Wingfield PT, Stahl SJ, Steven AC. Visualization of a 4-helixbundle in thehepatitis Bvirus capsid by cryo-electron microscopy. Nature. 1997;386:91-94.

[3] Matadeen R, Patwardhan A, Gowen B, Orlova EV, Pape T, Cuff M, Mueller F, Brimacombe R, van Heel M. The Escherichia coli large ribosomal subunit at 7.5 Å resolution. Struct Fold Des. 1997;7:1575-83.

[4] Zhou ZH, Baker ML, Jiang W, Dougherty M, Jakana J, Dong G., Lu G, Chiu W. Electron cryomicroscopy and bioinformatics suggest protein fold models for rice dwarf virus. Nat Struct Biol. 2001;8:868-73.

[5] Liu HR, Jin L, Koh SBS, Atanasov I, Schein S, Wu L, Zhou ZH. Atomic structure of human adenovirus by cryo-em reveals interactions among protein networds. Science. 2010;329:1038-43. PMid: 16516173.

[6] Ludtke SJ, Chen DH, Song JL, Chuang DT, Chiu W. Seeing GroEL at 6 Å resolution by single particle electron cryomicroscopy. Structure. 2004;12:1129-36.

[7] Crowther RA. Procedures for three-dimensional reconstruction of spherical viruses by fourier synthesis from electron micrographs. Philos Trans R Soc Lond B Biol Sci. 1971;261:221-30.

[8] Ludtke SJ, Baldwin PR, Chiu W, EMAN: semi-automated software for high resolution single particle reconstructions. J Struct Biol. 1999;128:82-97. PMid: 11584308.

[9] Grigorieff N. FREALIGN: high-resolution refinement of single particle structures. J Struct Biol. 2007;157:117-12. PMid: 17135471.

[10] Navaza J. On the three-dimensional reconstruction of icosahedral particles. J Struct Biol. 2003;144:13-23.

[11] Liu HR, Cheng LP, Zeng SJ, Cai CY, Zhou ZH, Yang QB. Symmetry-adapted spherical harmonics method for high-resolution 3D single-particle reconstructions. J Struct Biol. 2008;161:64-73. PMid: 18992072.

[12] Zeng SJ, Liu HR, Yang QB. Application of symmetry adapted function method for three-dimensional reconstruction of octahedral biological macromolecules. International Journal of Biomedical Imaging. 2010; 2010: Article ID. 195274:1-11. PMid: 9486647.

[13] Provencher SW, Vogel RH. Three-dimensional reconstruction from electron micrographs of disordered specimens. I. Method. Ultramicroscopy. 1988;25:209-22.

[14] Vogel RH, Provencher SW. Three-dimensional reconstruction from electron micrographs of disordered specimens II. Implementation and results. Ultramicroscopy. 1988;25:223-39. PMid: 9499710.

[15] Zheng Y, Doerschuk PC, Johnson JE. Determination of three dimensional low-resolution viral structure from solution X-ray scattering data. Biophys J. 1995;69:619-39.

[16] Alexander F, Cheng YF, Grigorieff N, Walz T. Structure of an auxilin-bound clathrin coat and its implications for the mechanism of uncoating. Nature. 2004;432:649-53.

[17] Ludtke SJ, Baker ML, Chen DH, Song JL, Chuang DT, Chiu W. De novo backbone trace of GroEL from single particle electron cryomicroscopy. Structure. 2008;16:441-48. PMid: 17765208.

[18] Rabl J, Smith DM, Yu YD, Chang SH, Goldberg AL, Cheng Y. F. Mechanism of gate opening in the 20S proteasome by the proteasomal ATPases. Mol Cell. 2008;116:565-76.

[19] Mouche F, Zhu YX, Pulokas J. Automated three-dimensional reconstruction of keyhole limpet hemocyanin type 1. J Struct Biol. 2003;144:301-12.

[20] Bradley, CJ, Cracknell, AP. The mathematical theory of symmetry in solid. Clarendon, UK: Oxford. 1972.

[21] Chen, JQ, Group representation theory for physicists. Singapore: World scientific.1989.

[22] Fernando GW, Watson RE, Davenport JW. Point group symmetries and gaussian integration. J Comput Phys. 1994;112:282-90. PMid: 14570167.

[23] Chen JQ, Fan PD. Algebraic solutions for all dihedral groups. J Math Phys. 2000;41:8196-222.

[24] Abramowitz, M, Stegun, IA. Handbook of mathematical functions. New York:Dover Press,1965. PMid: 17709239.

[25] Saxton WO, Baumeister W. The correlation averaging of a regularly arranged bacterial cell envelope protein. J Microsc. 1982;127:127-38. PMid: 769017.

[26] Harauz G, van Heel M. Exact filters for general geometry three-dimensional reconstruction. Optik. 1986;73:146-56. 